\title{
Estructuras de navegación para e-learning
}

\author{
Por Cristòfol Rovira
}

Se presenta una síntesis de las principales conclusiones y de los resultados obtenidos en la investigación realizada por Cristòfol Rovira (profesor de documentación de la Universidad Pompeu Fabra) con motivo de su tesis doctoral sobre enseñanza online de la documentación e hipertextos.

EN LOS ÚLTIMOS AÑOS DE LA década de los 90 y principios del nuevo milenio se está produciendo un cambio muy importante en la docencia universitaria provocado por la rápida convergencia entre 3 tipos de tecnologías: las telecomunicaciones, la informática y los medios audiovisuales.

En estos términos se pronuncia el informe CRE (Conflict resolution education) de la Assoc. of European Universities, abril de 1998, cuando propone estrategias para reestructurar la universidad aplicando las nuevas tecnologías a los procesos de enseñanza-aprendizaje. El elemento más visible de esta innovación tecnológica en la pedagogía universitaria es la creación de campus virtuales para la docen- cia en numerosas universidades. Se trata de espacios digitales compartidos, soportados con tecnología web, donde poder consultar información, comunicarse de manera asíncrona y realizar actividades de aprendizaje interactivas.

"Rapid convergence of hitherto separate technologies (e. g. telecommunications, computing, television and audiovisual) combined with falling unit cost of powerful networked devices (e. g. pcs with internet/intranet connections, network computers, other digital devices and consoles) are already changing the nature of work and organizations. Ver-
Cristòfol Rovira es doctor en ciencias de la educación, licenciado en filosofía y ciencias de la educación, ingeniero técnico en informática de gestión y diplomado en biblioteconomía y documentación. Es profesor de la Universidad Pompeu Fabra y director del Máster online en documentación digital.

http://www.hipertext.net

http://www.documentaciondigital.org http://www.mapasconceptuales.com

\begin{tabular}{|c|c|c|c|}
\hline & $\begin{array}{c}\text { Modelado } \\
\text { orientado a } \\
\text { objetos para un } \\
\text { programa } \\
\text { informático }\end{array}$ & $\begin{array}{l}\text { Modelado orientado a } \\
\text { objetos para un } \\
\text { hipertexto } \\
\text { (versión simple) }\end{array}$ & $\begin{array}{c}\text { Modelado orientado a objetos } \\
\text { para un hipertexto } \\
\text { (versión compleja) }\end{array}$ \\
\hline \multirow[t]{3}{*}{ Modelos } & $\begin{array}{l}\text { Modelo de } \\
\text { objetos }\end{array}$ & Modelo de objetos & Modelo de objetos \\
\hline & Modelo dinámico & - & - \\
\hline & Modelo funcional & - & - \\
\hline \multirow[t]{9}{*}{$\begin{array}{l}\text { Conceptos } \\
\text { básicos }\end{array}$} & $\begin{array}{l}\text { Objeto e } \\
\text { instancia }\end{array}$ & Nodo & Conjunto de nodos, nodo y fragmento de nodo \\
\hline & Clase & Tipo de nodo & Tipo de conjunto de nodos, nodo y fragmento de nodo \\
\hline & Atributo & $\begin{array}{c}\text { Característica del } \\
\text { nodo }\end{array}$ & Característica del conjunto de nodos, del nodo y del fragmento de nodo \\
\hline & Agregación & Agregación de nodos & Agregación de conjunto de nodos, nodo y fragmento de nodo \\
\hline & Enlace & Enlace hipertextual & Enlace hipertextual \\
\hline & Asociación & Tipo de enlace & Tipo de enlace \\
\hline & Método & - & - \\
\hline & $\begin{array}{c}\text { Herencia de } \\
\text { clases }\end{array}$ & $\begin{array}{c}\text { Herencia de tipos de } \\
\text { nodos }\end{array}$ & Herencia de tipos de conjunto de nodos, nodo y fragmento de nodo \\
\hline & Polimorfismo & - & - \\
\hline
\end{tabular}

Tabla 1. Modelado orientado a objetos aplicado a los hipertextos 
forma masiva en la educación (televisión educativa, medios audiovisuales, software educativo, sistemas expertos educativos, etc.). Aun así, internet tiene un coste económico muy reducido y unas potencialidades pedagógicas no disponibles hasta ahora. Integra en un solo medio la comunicación masiva, el acceso automatizado a la información (por navegación o por interrogación), características multimedia y la interacción propia del software educativo.

Estos factores hacen que, pese a los precedentes, se haya reformulado de nuevo una vieja utopía en la que el profesor concentrará sus esfuerzos realizando intervenciones "de calidad" en pequeños grupos de trabajo, con actividades académicas creativas, con tutorías flexibles y personalizadas, etc., mientras que la mera transmisión de información será realizada por sistemas docentes no presenciales residentes en los nuevos campus virtuales.

"The bottom-up perspective [la visión desde el Rectorado y los decanatos] is concerned with the potential for deploying technology to achieve and sustain real change, confronting such intractable issues as the tedium of

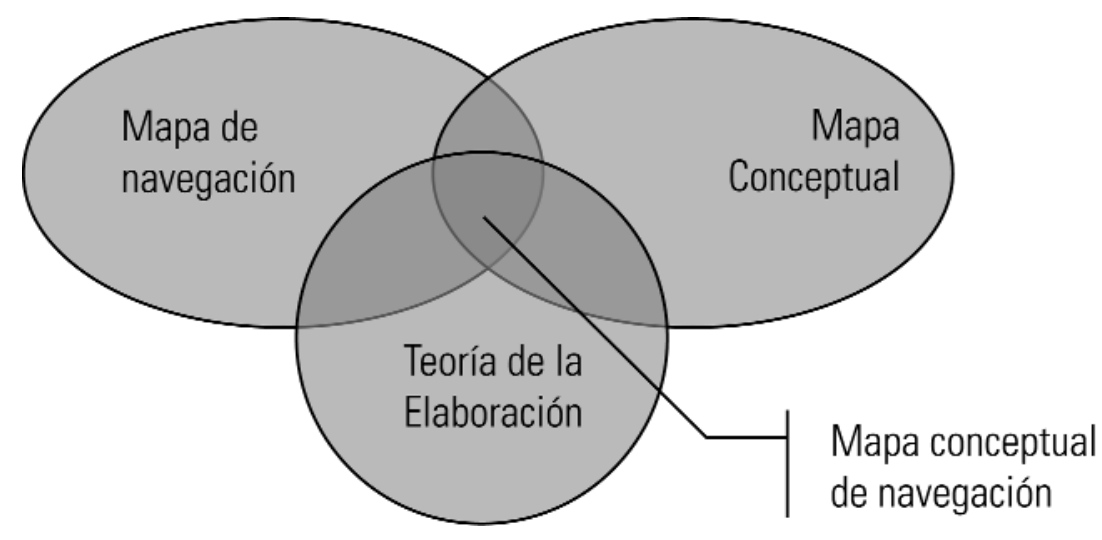

Figura 1. Bases teóricas del mapa conceptual de navegación the 'transcription' lecture, meaningful discourse with students in large class groups (on or off campus, synchronous or asynchronous), provision of improved tutorial support for assignment that are intellectually stimulating and challenging, and the provision of support for innovations such as collaborative working/peer tutoring". (Association of European Universities, 1998, pp. 10-11).

Este nuevo horizonte está sustentado sobre 2 propiedades, aparentemente heterogéneas, que la convergencia de las telecomunicaciones, la informática y los medios audiovisuales han hecho posible: la comunicación asíncrona y la organización hipertextual de la información.

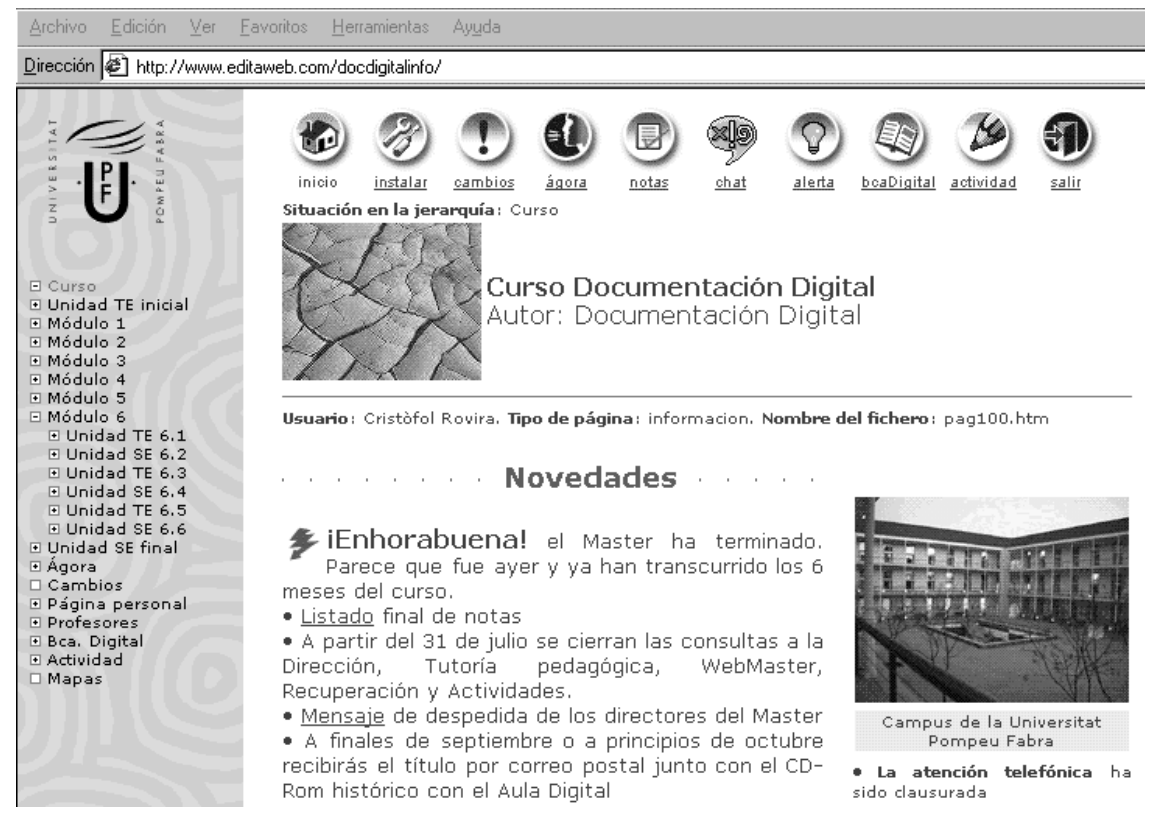

Figura 2. Página inicial del aula digital del Master online en documentación digital.
La segunda actúa como tejido conjuntivo uniendo espacios virtuales de comunicación (correo electrónico, foros de discusión, trabajo en grupo, etc.), con contenidos didácticos (documentos digita-

\section{«El informe CRE de abril de 1998 propo- ne estrategias para reestructurar la uni- versidad aplicando las nuevas tecnologí- as a los procesos de enseñanza-aprendi- zaje»}

les de varios tipos), software de autoaprendizaje (pruebas de elección múltiple, simulaciones interactivas, etc.) y sistemas de recuperación de información (motores de búsqueda y bases de datos).

En estos momentos, el grado de complejidad de los materiales digitales que contienden los campus virtuales es muy diverso. Podemos encontrar desde un texto secuencial (antes entregado a los estudiantes en fotocopias), hasta complejos documentos multimedia con un alto grado de interacción. No obstante, la corta pero intensa evolución en el diseño de estos espacios digitales apunta hacia un tipo de material didáctico, cuya novedad residirá en la explotación con finalidades pedagógicas de las posibilidades que ofrece el hiper- 
texto para la organización flexible de la información.

Desde una perspectiva pedagógica, el diseño de los materiales didácticos hipertextuales replantea, en un nuevo contexto, un problema clásico de las ciencias de la educación sobre la organización óptima de los contenidos educativos para favorecer la enseñanza-aprendizaje. Al mismo tiempo, desde la perspectiva de las ciencias de la documentación, se hace evidente la necesidad de adaptar el diseño genérico de la navegación hipertextual a las exigencias específicas del hiperdocumento cuando actúa como material didáctico. El objetivo de facilitar el aprendizaje (organización y secuenciación de los contenidos) ha de integrarse con la posibilidad de facilitar el acceso a la información (diseño de navegación) para crear materiales didácticos en formato hipertextual eficientes.

Por tanto, parece necesario (y urgente) encontrar las bases de un conjunto de principios que puedan guiar el diseño y la creación de materiales didácticos hipertextuales. Su búsqueda ha sido, precisamente, el motivo de realización de la tesis doctoral que reseñamos en este artículo. Estos principios tendrían que ser sólidos para permitir a los responsables académicos trabajar con un mínimo de confianza respecto a su eficiencia; pero también tendrían que ser flexibles para poder adaptarse a diferentes entornos y situaciones didácticas, necesidades pedagógicas, etc.

En la investigación hemos partido de la presuposición de que una parte importante de los principios que rigen el diseño de cualquier tipo de hipertexto están relacionados con el conjunto de herramientas de ayuda a la navegación, lectura y consulta de los hiperdocumentos. A su vez, los sistemas o herramientas de navegación están basados en las estructuras de información que forman los enlaces del hi- pertexto al conectar los diferentes nodos o fragmentos independientes de información (Codina, 2000; Nielsen, 2000; Rosenfeld y Morville, 1998; Lynch y Horton, 1999).

Hay un importante consenso entre estos autores en cuanto que los sumarios, índices y mapas o barras de navegación forman la parte fundamental del sistema de navegación de un hipertexto. En este sentido, Resenfeld y Morville (1998), autores de una de las obras más influyentes sobre representación y organización de la información en formato hipertextual indican lo siguiente:

"Navigation systems are composed of a variety of elements. Some, such as graphical navigation bars and pop-up menus, are implemented on the content-bearing pages themselves, others, such as tables of contents o site maps, provide remote access to content within the organization structure. While these elements may be implemented on each page, together they make up a navigation system that has important site-wide implications. A well-designed navigation system is a critical factor in determining the success of your web site" (Rosenfeld; Morville, 1998, p. 48).

Las estructuras de conexiones entre enlaces constituyen la arquitectura de los posibles caminos de lectura (o estructura de navegación) de un hipertexto y establecen las posibilidades de interacción del lector.

"Web sites are build around basic structural themes. These fundamental architectures govern the navigational interface of the web site and mold the user's mental models of how the information is organized. Four essential structures can be used to build a web site: sequence, grids, hierarchies, and webs" (Lynch y Horton, 1999, pp. 27).

Sintetizando las aportaciones básicas de los autores mencionados, las estructuras básicas son: secuencia, secuencia con alternativas, jerarquía y red (Codina, 1996, p. 109; Rosenfeld y Morville, pp. 26-46; Lynch y Horton, 1999, pp. 24-30). Los sistemas y las estructuras de navegación son los principales elementos de un hipertexto para proporcionar al lector el soporte indispensable que necesitará para su consulta. Nielsen (2000) reclama este apoyo a la navegación para la web de internet.

\section{«La flexibilidad en la organización de la in- formación que ofrece el hipertexto abre nuevas posibilidades que es necesario ex- plorar»}

"The web is a navigational system: the basic user interaction is to click on hypertext links in order to move around a huge information space width hundreds of millions of pages. Because the space is so bast, navigation is difficult, and it becomes necessary to provide users width navigational support beyond the simple 'go-to' hyperlinks” (Nielsen, 2000, p. 188).

\section{Propósito del estudio}

En cualquier caso, y pese a la abundante bibliografía (sobre todo técnica), sobre estos temas, hay pocos estudios en profundidad sobre cuáles son los principios del diseño de los sistemas de la navegación y todavía menos sobre cómo aplicarlos al campo de la enseñanzaaprendizaje. Aun así, la estructura de la información es un factor esencial para conseguir que un ma- 
terial didáctico, hipertextual o no, sea efectivo.

La importancia de la forma de organizar la información está justificada porque en el contexto del hipertexto para la enseñanza-aprendizaje, se identifica completamente con la organización de los contenidos didácticos. Del mismo modo que esto último es básico por favorecer o dificultar el proceso de enseñanza-aprendizaje (Briggs, 1973), el diseño de navegación será también primordial cuando se realice por medio de un hipertexto. Estas reflexiones delimitan el objeto de estudio de la tesis que quedó formulado de la siguiente manera: "El diseño de navegación y la organización de los contenidos en el hipertexto para la enseñanzaaprendizaje".

\section{Objetivos}

Se pueden resumir de la siguiente manera:

-Determinar las propiedades y características del objeto de estudio "el hipertexto para la enseñanza-aprendizaje".

—Establecer los principios básicos del diseño de navegación y la organización de los contenidos para conseguir efectividad, tanto desde una perspectiva pedagógica como documental.

- Proponer un análisis del hipertexto donde los puntos de vista pedagógico y documental se fusionen y la estructura de la información se identifique con la organización de los contenidos didácticos.

- Proponer un modelo del proceso para la creación del hipertexto que fusione la estructuración de la información con la organización de contenidos.

-El punto anterior permitiría establecer una metodología de análisis y diseño de hipertextos para la enseñanza-aprendizaje que combi- ne las perspectivas pedagógica y documental.

- Presentar nuevas hipótesis bien fundamentadas por tratar de determinar la dirección de futuras investigaciones para la elaboración de una teoría más general sobre el diseño de la navegación del hipertexto para la enseñanza-aprendizaje.

Esta nueva teoría tendría que ser de carácter interdisciplinar, e integraría bases teóricas procedentes de al menos 3 disciplinas diferentes: pedagogía, documentación y ingeniería del software. Así pues, la tesis es el intento de presentar los primeros fundamentos teóricos de una nueva línea de investigación, enmarcada en los objetivos generales planteados y a partir del desarrollo de 2 líneas de trabajo que constituyen su núcleo central: delimitar el campo teórico interdisciplinar donde encontrar las bases conceptuales que permitan lograr los objetivos planteados y la realización de un estudio de caso exploratorio sobre un ejemplo concreto de concepción, diseño, realización y utilización de un material didáctico en formato hipertextual.

\section{Metodología de investigación e hipótesis}

Como consecuencia de lo indicado en el punto anterior, las disciplinas implicadas son:

- La didáctica, la teoría del aprendizaje, el diseño de instrucción y las teorías de la organización de contenidos (ciencias de la educación).

- La teoría del hipertexto, el diseño de la navegación hipertextual y el análisis y diseño de hiperdocumentos con tecnología web (ciencias de la documentación).

—Los métodos de modelado y representación de los sistemas de información y las metodologías para su análisis y diseño (ingeniería del software).
El estudio teórico se concretó realizando un estado de la cuestión que incluyó la organización de los contenidos didácticos en relación al hipertexto, la teoría del hipertexto, la teoría del hipertexto para la enseñanza-aprendizaje y las metodologías de diseño de la ingeniería del software.

Por otro lado, el estudio de caso se llevó a cabo sobre 3 ediciones sucesivas de un curso de postgrado impartido totalmente a través de internet y diseñado de acuerdo con los principios discutidos y presentados en este trabajo (Curso y diploma de postgrado en documentación digital, ediciones 1998/1999, 1999/2000 y 2000/2001), con los siguientes objetivos:

-Describir las características y la evolución de las 3 ediciones de dicho curso.

-Identificar las bases teóricas más pertinentes al objeto de estudio, contrastándolas con el contexto empírico de docencia.

-Obtener las primeras evidencias, basadas en el caso investigado, que permitieron emitir juicios razonables y razonados sobre la interrelación entre los diversos tipos de organización de los contenidos didácticos que permite el hipertexto y el tipo de resultados de aprendizaje.

-Formular hipótesis bien fundamentadas para determinar la dirección de futuras investigaciones para la creación de una teoría sobre el diseño de la navegación en el hipertexto para la enseñanza-aprendizaje.

Las hipótesis de trabajo que nos planteamos con el fin de guiar el estudio teórico y el estudio de caso fueron las siguientes:

Hipótesis 1: hay una interrelación entre la estructura de la información de los materiales didácticos en formato hipertextual y el tipo de resultados de aprendizaje, de 
manera que determinadas estructuras facilitan más unas determinadas formas de enseñanza-aprendizaje que otras.

Hipótesis 2: los hipertextos basados en tecnología web pueden proporcionar el contexto lógico y tecnológico que permita unificar los elementos heterogéneos de información, comunicación y de interacción que constituyen un material didáctico en formato digital.

Hipótesis 3: es posible identificar y definir un conjunto de principios básicos de diseño de hipertextos donde se integren la navega-

\section{«Parece urgente en- contrar las bases de un conjunto de prin- cipios que puedan guiar el diseño y la creación de materia- les didácticos hiper- textuales»}

ción hipertextual y la estructuración de la información con la organización y la representación de los contenidos didácticos.

Los principales resultados de la investigación realizada pueden resumirse en 3 apartados. En primer lugar la formulación de 7 principios generales para el diseño de navegación hipertextual aplicables en la creación de aulas digitales. En segundo lugar, la propuesta de Maidhea (Modelado abstracto interdisciplinar del hipertexto para la enseñanza-aprendizaje), un método de modelado del hipertexto que facilita su diseño y desarrollo, resultando ser especialmente indicado para los materiales didácticos hipertextuales. Finalmente también se obtuvo un resultado inesperado: los mapas conceptuales de navegación, una nueva herramienta de ayuda a la navegación basada en las relaciones semánticas de los contenidos.

\section{Principios}

Como ya hemos indicado, el resultado de la realización del estudio de caso fueron los 7 principios que a continuación presentamos, aplicables a procesos de enseñanza-aprendizaje impartidos a través de internet con cualquier aula digital construida con tecnología web:

1. Interrelación entre estructuras y resultados de aprendizaje. Hay una correspondencia entre la estructura de la información de los materiales didácticos en formato hipertextual y el tipo de resultados de aprendizaje, de modo que la estructura secuencial de los nodos de información de un hipertexto es adecuada para la enseñanza-aprendizaje inicial de una materia o área de conocimiento, en cambio la estructura en red es más adecuada para la enseñanza-aprendizaje avanzada.

2. Integración de estructuras. Un diseño de la estructura de navegación con las siguientes características será adecuado, tanto para estudiantes neófitos como para los más avanzados:

a. El contenido se deberá estructurar con un nodo central extenso, con los materiales básicos y un conjunto de nodos de corta extensión con los complementarios y avanzados.

b. La estructura del nodo central deberá ser secuencial siguiendo el formato clásico de un artículo en papel, con introducción, desarrollo y conclusiones, pero con enlaces hacia nodos complementarios que permitan ampliar los contenidos según las decisiones que adopte el alumno.

c. El nodo central deberá estar enlazado con los complementarios, los cuales formarán entre ellos una estructura en red.

3. Permanencia de la representación jerárquica. La consulta o navegación de un hipertexto para la enseñanza-aprendizaje mejorará cuando la estructura jerárquica académica implícita en los materiales didácticos se haga explícita en un sumario jerárquico con las siguientes características:

a. Deberá contener todas las páginas o nodos del hipertexto.

b. Formará parte de todas las páginas y no será un elemento independiente.

c. El ítem de la página activa del sumario y la ruta para llegar a ella deberán quedar marcados con elementos gráficos haciendo explícito el sitio que ocupa en la jerarquía general del hipertexto.

4. Mapa conceptual de navegación. La consulta o navegación de un hipertexto y el aprendizaje de sus contenidos mejorará cuando las relaciones conceptuales entre éstos se representen con mapas conceptuales de navegación que tengan las siguientes características:

a. Deberá estar formado por un conjunto de conceptos organizados jerárquicamente y representados de manera gráfica por medio de relaciones etiquetadas con palabras enlace.

b. Cada concepto que forme parte del mapa estará asociado a un listado de enlaces hipertextuales que permitan el acceso a los nodos o páginas donde sea desarrollado.

5. Fragmentación temática. La consulta o navegación de un hipertexto y el aprendizaje de sus contenidos mejorará cuando éstos se encuentren fragmentados de acuerdo con criterios temáticos y de la siguiente manera:

a. Deberán dividirse en unidades didácticas de entre 0,5 a 1 crédito que después se agruparán en módulos que variarán de 3 a 6 créditos.

b. Cada unidad didáctica deberá contener un artículo central con 
alrededor de 3.000 a 7.500 palabras y varias páginas complementarias de extensión reducida.

c. Cada módulo agrupará entre 6 y 8 unidades didácticas.

6. Doble representación conceptual. La consulta o navegación de un hipertexto y el aprendizaje de sus contenidos mejorará cuando cada unidad o módulo contenga 2 mapas conceptuales de navegación, de acuerdo con las siguientes características:

a. Cada unidad didáctica deberá contener un mapa conceptual de navegación inicial y otro final. El primero cumplirá una función de epítome inicial y contendrá sólo los conceptos esenciales de la unidad didáctica. En el segundo caso contendrá todos los conceptos importantes y se construirá complementando los conceptos, relaciones y palabras enlace que hay en el mapa inicial.

b. Cada módulo deberá contener un mapa conceptual de navegación inicial y otro final siguiendo las mismas características que los anteriores, pero a un nivel jerárquico superior.

7. Redundancia. El diseño de navegación de un hipertexto mejora cuando hay redundancia en las opciones de navegación de manera que para establecer una ruta de navegación hay siempre 2 o más posibilidades disponibles.

\section{Mapas conceptuales de navegación}

Partiendo de la teoría de la elaboración (Reigeluth, 1983, 1999), y de acuerdo con el principio sexto, la investigación desarrollada en esta tesis proponeun nuevo tipo de sistema de navegación denominado «Mapa conceptual de navegación» con las siguientes características:

-Contiene la representación gráfica de la estructura conceptual de los contenidos de un material didáctico hipertextual.

- Sigue el esquema clásico de un mapa conceptual formado por un conjunto de conceptos organizados jerárquicamente y representados de manera gráfica por medio de relaciones etiquetadas con palabras enlace.

-Es un mapa de navegación porque los conceptos tienen asociado un listado de enlaces hipertextuales que permiten el acceso a los nodos o páginas en los que el concepto se desarrolla.

\section{«En el contexto del hipertexto para la en- señanza-aprendizaje, la estructura de la in- formación se identifi- ca completamente con la organización de los contenidos di- dácticos»}

La propuesta de esta nueva herramienta de navegación ha sido desarrollada en posteriores trabajos dando como resultado un editor online para la creación y la modificación de mapas conceptuales de navegación. Un editor que los guarda en formato topic map y que está disponible para ser utilizado en línea.

\section{http://www.mapasconceptuales.com}

Otro de los resultados de la investigación es el ya citado Maidhea, un método para representar gráficamente cualquier hipertexto para la enseñanzaaprendizaje (figura 1). Integra las siguientes disciplinas:

—Ciencias de la educación con los mecanismos de planificación didáctica, las teorías de la organización del contenido y concretamente la teoría de la elaboración.
- Ciencias de la documentación con los métodos y modelos de diseño de estructuras y sistemas de navegación hipertextual.

- Teoría del hipertexto para la enseñanza-aprendizaje con la $\operatorname{cog}$ nitive flexibility theory (Spiro, 1990; Spiro, 1991).

-Ingeniería del software y los métodos de representación de sistemas de información orientados a objetos.

El principal objetivo de Maidhea es representar los materiales didácticos hipertextuales, y el hipertexto en general, con diagramas con la finalidad de facilitar la comunicación entre equipos interdisciplinares que participan en su desarrollo y posterior mantenimiento. Un segundo objetivo es representar con la misma notación, y de forma integrada, la planificación didáctica, la organización de los contenidos didácticos, el diseño de sistemas de navegación y la estructuración de la información del hipertexto. Finalmente, también se plantea representar modelos genéricos de hipertextos que podrían aplicarse como plantillas para la creación de nuevos materiales didácticos hipertextuales.

La formulación básica del modelado Maidhea proviene de la orientación a objetos, de manera que los materiales didácticos hipertextuales, son considerados sistemas de información organizados en colecciones de objetos, los cuales tienen unas estructuras de datos con sus atributos o propiedades.

Por otro lado, integra la mayoría de los elementos que de manera explícita o implícita están presentes en las propuestas metodológicas clásicas sobre el diseño y modelado de hipertextos como Hypertext abstract machine (HAM) (Campbell, 1988), Hypertext design model (HDM) (Garzotto, 1990), Lange (1994), Relationship management methodology (RMM) 
(Isakowitz, 1995) y Object oriented hypermedia design methodology (Oohdm) (Schwabe, 1996). Estos elementos son:

—Definición de diferentes perspectivas para observar y después representar el hipertexto.

-Representación gráfica de las diferentes perspectivas formando diversos modelos interconectados del hipertexto.

- Clara separación entre una fase de diseño y una de implementación en el proceso de creación.

-Establecimiento de tipos y de instancias de elementos en el contexto del paradigma de la orientación a objetos de la ingeniería del software.

En Maidhea se aplica la notación de los diagramas de clases de la OMT (Object modeling technique) por razones de facilidad de uso. No obstante, se podrían utilizar con la misma efectividad otros sistemas de representación del análisis de orientación a objetos, como el OML (Open modeling language) o el UML (Unified modeling language).

El principio primordial de la utilización del modelado orientado a objetos al diseño de materiales didácticos hipertextuales es considerar que cada nodo o página de un hiperdocumento es una instancia de un objeto con sus atributos o características y que esta instancia de página pertenece a una «clase» genérica de páginas.

También son objetos conjuntos de diversas páginas y fragmentos de páginas que tienen entidad propia formando parte de otras muchas. Por lo tanto, las clases en Maidhea son 3: nas.

-Representan tipos de pági-

- Simbolizan tipos de conjuntos de páginas: unidades didácticas, módulos y el propio curso.
- Representan tipos de fragmentos de páginas, como párrafos, elementos de navegación, menús desplegables, el menú gráfico superior, el mapa conceptual, etc.

Los atributos o características de las clases permiten almacenar de forma ordenada todos los datos significativos para la planificación didáctica, el diseño de navegación e incluso el diseño gráfico. Se pueden clasificar en 4 tipos según sus propiedades:

-Para identificar la clase: título, identificador, fecha de creación, etc.

- Características de la planificación didáctica: objetivos didácticos, actividades de aprendizaje, temporalización, etc.

-Propiedades del diseño de navegación: padre e hijo en el sumario jerárquico general, opciones de los sumarios, identificador de la página inicial, nivel jerárquico de la página, etc.

-Peculiaridades del diseño gráfico: color, tramas gráficas para el fondo de las páginas, distribución de los elementos, etc.

\section{«Maidhea es un mé- todo para representar gráficamente cual- quier hipertexto para la enseñanza-apren- dizaje»}

La «relación de agregación» de la orientación a objetos permite representar las relaciones entre el todo y sus partes en los objetos de los materiales didácticos hipertextuales. En cuanto a la «relación de generalización» hace posible la representación de las relaciones de tipo padre-hijo entre un objeto y las versiones más refinadas de este mismo objeto que asumen, por «herencia», los atributos de la clase padre. Las clases abstractas son, acorde con la $O M T$, las que no tienen instancias y permiten repre- sentar relaciones de generalización entre entidades con características comunes.

Como podemos ver en la tabla 1 , solamente se utiliza una parte de los elementos que forman el modelado orientado a objetos. Los diagramas que representan hiperdocumentos no incluyen el software que soporta la funcionalidad del hipertexto, sino que muestran sólo el diseño externo centrado en la fragmentación, estructuración y organización de la información en el material didáctico hipertextual. Por lo tanto, los elementos de la orientación a objetos relacionados directamente con la representación de esta funcionalidad, como los métodos, no se utilizan en Maidhea. Los símbolos que se han utilizado en la representación del modelado Maidhea son los estándares según la $O M T$, añadiendo únicamente el enlace hipertextual direccional como un tipo más de relación entre clases de objetos o entre instancias de objeto.

\section{La obra}

La tesis doctoral de la que damos cuenta en este artículo, fue presentada en diciembre de 2001 en la Universidad de Barcelona con el título, El disseny de navegació $i$ l'organització de continguts en l'hipertext per a l'ensenyament-aprenentatge. Fue dirigida por los doctores Lluís Codina y Miguel Ángel Aguareles, logrando la máxima calificación.

Entre otros aspectos, se analizó el diseño de navegación del aula digital durante las 3 primeras ediciones del Curso de postgrado en documentación digital (actualmente Master online en documentación digital), impartido de manera no presencial y a través de internet por la Universidad Pompeu Fabra de Barcelona. El autor quiere agradecer los comentarios y sugerencias de los estudiantes de estas 3 prime- 
ras ediciones que contribuyeron en la realización de la tesis.

http://docdigital.upf.es

\section{Bibliografía}

Aguareles, Miguel Ángel. «Educación y documentación». En: Baró, Jaume; Cid, Pilar (eds.). Anuari Socadi de documentació i informació. Barcelona: Socadi, 1998.

Association of European Universities. "Restructuring the university. New technologies for teaching and learning. Guidance to universities on strategyÅh. En: CRE (Conflict resolution education) guide, 1998, April, n. 1.

http://www.unige.ch/cre/publications/docs $\% 20 p$ df/CREguidel.pdf

Association of European Universities. ÅgRestructuring the university. Universities and the challenge of new technologies Åh. En: CRE doc, 1996, November, n. 1.

http://www.unige.ch/cre/publications/docs $\% 20 p$ df/CREdocl.pdf

Briggs, L. J. ÅgEl ordenamiento de secuencia en la instrucciónÅh. Buenos Aires: Guadalupe, 1973.

Campbell, Brad; Goodman, Joseph M. «Anzuelo: a general purpose hypertext abstract machine». En: ACM, 1988, July, v. 31, n. 7.

Codina, Lluís. El libro digital y la www. Madrid: Tauro Pro, 2000

Garzotto, Franca; Paolini, Paolo; Schwabe, Daniel. «HDM-a model-based approach to hypertext aplication design». En: ACM transactions on Information Systems, 1993, July, v. 11, n. 1.

Hoffman, Suzanne. «Elaboration theory and hypermedia: is there a link?». En: Educational technology, 1997, enero-febrero.

Isakowitz, Tomás; Stohr, Edward A.; Balasubramaian, P. Narasimhan. «RMM: a met- hodology for structured hypermedia design». En: Communications of the ACM, 1995, v. 38 , n. 8 .

Lange, Danny B. An object-oriented design method for hypermedia information systems. Twenty-seventh annual Hawai international conference on systems sciences, 1994.

Lynch, P.; Horton, S. Web style guide. Basic design principles for creating web sites. Yale University Press, 1999.

Nielsen, Jakob. Designing web usability. Indianapolis: New Riders, 2000.

Nielsen, Jakob. The Alertbox: current issues in web usability, 1998. Consultado en: octubre de 2002.

http://www.useit.com/alertbox/

Nielsen, Jakob. «Avaluating hypertext usability». En: Jonassen, D. H.; Mandl, H. (eds.) Designing hypermedia for learning. Berlin: Springer-Verlag, 1990.

Reigeluth, Charles M. (ed.). Instructional-design theories and models. Volume II. A new paradigm of instructional theory. NJ: Mahwah, London: Lawrence Erlbaum, 1999.

Reigeluth, Charles M.; Stein, Faith S. «The elaboration theory of instruction». En: Reigeluth, C. M. (comp.). Instructional design theories and models: an overview of their current status. Hillsdale: Erlbaum, 1983.

Rosenfeld, L.; Morville, P. Information architecture for the world wide web. Cambridge: O’Reilly, 1998.

Rovira, Cristòfol. «La intranet documental: una eina per a la docència en documentació». En: 6es. Jornades catalanes de documentació, 1997.

Rovira, Cristòfol. «El hipertexto la recuperación de informació en la Web». En: Baró, Jaume (ed.). Buscar y colocar información en el world wide web. Barcelona: Libros del índice, 1998.
Rovira, Cristòfol. ÅgLa orientación a objetos en el diseño de hipertextos para la enseñanzaaprendizajeÅh. En: Cuadernos de documentación multimedia, 1999, n. 8

Rovira, Cristòfol. "Documents hipertextuals per a entorns virtuals d'aprenentatge". En: $D i$ git.hvm. Revista digital d'humanitats, v. 1. Consultado en: 10-04-02.

http://www.uoc.edu/humfil/digithum/digithuml/ crovira/croviraf.htm

Rovira, Cristòfol; Codina, Lluís (dirs.). Documentación digital, [cd-rom], 2000, v. 3.

Rovira, Cristòfol. El disseny de navegació $i$ l'organització de continguts en l' hipertext per a l'ensenyament-aprenentatge (tesis doctoral) Barcelona: Universitat de Barcelona, 2001.

Rumbaugh, James, et al. Modelado y diseño orientado a objetos. Madrid: Prentice Hall, 1996 y 1998.

Schwabe, Daniel; Rossi, Gustavo; Barbosa, Simone D. J. «Systematic hypermedia application design with Oohdm». En: ACM international conference on hypertext (Hypertext '96). New York: ACM, pp. 116-128, 1996. Isbn: 0 897917782

Spiro, Rand J., et al. «Cognitive flexibility, constructivism, and hypertext: random access instruction for advanced knowledge acquisition in ill-structured domains». En: Educational technology, 1991, v. XXXI, n. 5, pp. 24-33.

Spiro, Rand J.; Jehng, Jihn-Chang. «Cognitive flexibility and hypertext: theory and technology for the nonlinear and multidimensional transversal of complex subject matter». En: Don Nix; Rand J. Spiro. (eds). Cognition, education, and multimedia: exploring ideas in high technology. Hillsdale, NJ: Lawrence Erlbaum Associates, 1990

Cristòfol Rovira, Universidad Pompeu Fabra.

cristofol.rovira@cpis.upf.es 